\title{
Global stability of two-species mutualism model with proportional harvesting
}

\author{
Rusliza Ahmad* \\ Faculty of Computer and Mathematical Sciences, Universiti Teknologi MARA, Perak Branch, Tapah Campus, 35400 Tapah, Perak, \\ Malaysia
}

\section{A R T I C L E I N F O}

\section{Article history:}

Received 2 March 2017

Received in revised form

28 May 2017

Accepted 7 June 2017

\section{Keywords:}

Global stability

Lyapunov function

Mutualism model

Proportional harvesting

\begin{abstract}
A B S T R A C T
This paper deals with the study on a mathematical model consisting of mutualistic interactions among two-species with proportional harvesting. Harvesting function is introduced to describe the rate of removal of the species. The local stability analysis shows that the unique positive equilibrium point is asymptotically stable when certain conditions are satisfied. Global stability is discussed by constructing Lyapunov function. It has been shown that the unique positive equilibrium point is globally asymptotically stable. Finally, numerical simulations supporting theoretical results are also included.
\end{abstract}

(C) 2017 The Authors. Published by IASE. This is an open access article under the CC BY-NC-ND license (http://creativecommons.org/licenses/by-nc-nd/4.0/).

\section{Introduction}

Mutualism is an interaction between two species where both species benefit from the interaction (Rockwood, 2015). Some common examples of twospecies mutualism include zebra and wildebeest (Fay and Greeff, 2006), yucca moths and yucca plants (Starr et al., 2015) and damselfish and sea anemone (Benz, 2000). In a thorough review of the natural history of mutualisms, Janzen (1985) has argued that most mutualisms can be classified into four types; seed dispersal mutualisms, pollination mutualisms, digestive mutualisms and protection mutualisms. Some example of each type of mutualisms can be seen in Kot (2001). In general, mutualism may be facultative or obligate (Morin, 2011). In the case of facultative mutualism, each species is able to survive in the absence of the other. Zebra and wildebeest, damselfish and sea anemones are examples of facultative mutualism. A mutualism is obligate when one species cannot live without the other species. Yucca moths and yucca plants have a reciprocal obligate relationship.

One of the important issues in a mutualism system is global stability. Some biologists believe that local asymptotically stable equilibrium point is globally asymptotically stable in an ecological model (Cheng et al., 1981). A general method to establish global stability in a mutualism system is by

\section{* Corresponding Author.}

Email Address: rusliza259@perak.uitm.edu.my https://doi.org/10.21833/ijaas.2017.07.011

2313-626X/C) 2017 The Authors. Published by IASE. This is an open access article under the CC BY-NC-ND license (http://creativecommons.org/licenses/by-nc-nd/4.0/) constructing a Lyapunov function. Two different forms of Lyapunov functions have been constructed by Goh (1979) and Reddy et al. (2011) to show that the unique positive equilibrium point is globally asymptotically stable in a same basic model of mutualism.

Recently, Georgescu et al. (2016) have analyzed the dynamics of three models of mutualism and establishing the global stability via the method of Lyapunov functional. The authors found that the use of higher-order self- limiting terms cures the shortcomings of Lotka-Volterra mutualisms, preventing unbounded growth and promoting global stability.

In the context of mutualism interaction, some studies consider the effect of harvesting and time delay. The removal of a number of a species of population from its habitat is known as harvesting. According to Ouncharoen et al. (2012), harvest management is used to control increasing population and to meet the public demands for animal damage control, recreation or commercial harvesting. A delay is a general concept that can represent different phenomena such as the finite gestation period or maturation period of a species. For animals, the delay time may be the time it takes for an egg to develop into a fertile adult (Haberman, 1998). The global stability of the unique positive equilibrium point of mutualism model subject to proportional harvesting has been studied by León (2012). He constructed more than one Lyapunov function to prove the global stability of the same model.

The global stability analysis of mutualism model with time delay has been conducted by some 
researchers. Hsu and Ho (2006) obtained the conditions for global stability of two-species mutualism model with single delay by constructing a Lyapunov function. The authors illustrate their results by some example. The global stability analysis of mutualism models with two delays or without delays by constructing a Lyapunov function has been studied by León (2015). He found that if all the delayed feedback is positive then the stability properties are independent of the values of the delays. Saito (2002) derived necessary and sufficient conditions for the global stability of mutualism model with time delays in some specific cases using the method of Lyapunov function.

In this paper, we extend the work done by Goh (1979) and Reddy et al. (2011) by constructing a suitable Lyapunov function to prove the global stability of the unique positive equilibrium point of a mutualism model with proportional harvesting and illustrate our results by a numerical example. This model is different to a model appearing in León (2012). In particular, the mathematical analysis is simulated using the data for the population of wildebeest and zebra as reported in Fay and Greeff (2006).

\section{Lyapunov's second method}

Lyapunov's second method or direct method uses an energy-like function called the Lyapunov function to study the behavior of dynamical systems analytically (Do and Pan, 2009). This method is referred to as a direct method because no knowledge of the solution of the system of differential equations is required. Lyapunov's second method enables the analysis to be extended beyond a small region near the equilibrium point (global analysis). The basic idea of this technique for verifying stability is to seek an aggregated summarizing function that continually decreases towards a minimum as the system evolves. The following Definition 1 and Theorem 1 can be obtained from Boyce and DiPrima (1992).

Definition 1: Suppose that $\left(x_{0}, y_{0}\right)$ is an equilibrium point of a given nonlinear system

$$
\begin{aligned}
& \frac{d x}{d t}=f(x, y), \\
& \frac{d y}{d t}=g(x, y) .
\end{aligned}
$$

A function $V(x, y)$ defined on a region $\Omega$ of the state space and containing $\left(x_{0}, y_{0}\right)$ is a Lyapunov function if it satisfies the following three requirements:

1. $V(x, y)$ is continuous and has continuous first partial derivatives.

2. $V(x, y)$ is positive definite.

3. $\dot{V}(x, y)=\frac{\partial V}{\partial x} \frac{d x}{d t}+\frac{\partial V}{\partial y} \frac{d y}{d t}$ is negative semi definite.

Theorem 1: If there exists a Lyapunov function, then the equilibrium point is stable. If furthermore, the function is strictly negative for every point then the stability is asymptotic.

\section{Mathematical model}

We consider the mutualism model with proportional harvesting as follows

$\frac{d x}{d t}=r x-b x^{2}+\alpha x y-h_{x} x$,

$\frac{d y}{d t}=s y-e y^{2}+\beta x y-h_{y} y$,

where, $b=\frac{r}{K_{x}}$ and $e=\frac{s}{K_{y}}$. The symbols $x$ and $y$ denote the population size of the first species and the second species respectively, $r$ and $s$ are the intrinsic growth rates, $K_{x}$ and $K_{y}$ are the carrying capacities, $\alpha$ and $\beta$ measure the mutualism effect of $y$ on $x$ and $x$ on $y$ respectively, $h_{x} x$ and $h_{y} y$ are harvesting terms proportional to the respective population size and all the parameters are positive constants. Let $r_{1}=r-h_{x}$ and $s_{1}=s-h_{y}$, so model (1) can be written as follow

$\frac{d x}{d t}=r_{1} x-b x^{2}+\alpha x y$
$\frac{d y}{d t}=s_{1} y-e y^{2}+\beta x y$

Under the assumptions $r_{1}>0$ and $s_{1}>0$, model (2) have four equilibrium points; $E_{1}=(0,0), E_{2}=$ $\left(\frac{r_{1}}{b}, 0\right), \quad E_{3}=\left(0, \frac{s_{1}}{e}\right) \quad$ and $\quad E^{*}=$ $\left(x^{*}, y^{*}\right)=\left(\frac{e r_{1}+\alpha s_{1}}{b e-\alpha \beta}, \frac{b s_{1}+\beta r_{1}}{b e-\alpha \beta}\right) . E^{*}=\left(x^{*}, y^{*}\right)$ is the unique positive equilibrium point of the system (2) if $r_{1}=$ $r-h_{x}>0, s_{1}=s-h_{y}>0$ and $b e-\alpha \beta>0$. In this case, the range of harvesting level for model (2) are $0<h_{x}<r$ and $0<h_{y}<s$.

\section{Local stability}

In this section, the local stability analysis of equilibrium points of model (2) is investigated. In order to determine the stability of each equilibrium point, we need to compute the Jacobian matrix of model (2). The sign of the real part of the eigenvalues of this matrix evaluated at these equilibrium points determines its stability. The Jacobian matrix of model (2) takes the form

$J=\left(\begin{array}{cc}r_{1}-2 b x+\alpha y & \alpha x \\ \beta y & s_{1}-2 e y+\beta x\end{array}\right)$.

The Jacobian matrix of model (2) at $E_{1}$ is

$J_{1}=\left(\begin{array}{cc}r_{1} & 0 \\ 0 & s_{1}\end{array}\right)$.

The eigenvalues $\lambda_{1}=r_{1}, \lambda_{2}=s_{1}$ of $J_{1}$ are both positive. Thus, the equilibrium point $E_{1}$ of model (2) is unstable. The Jacobian matrix of the model (2) at $\mathrm{E}_{2}$ is

$J_{2}=\left(\begin{array}{cc}-r_{1} & \frac{\alpha r_{1}}{b} \\ 0 & \frac{b s_{1}+\beta r_{1}}{b}\end{array}\right)$. 
The eigenvalues of $\mathrm{J}_{2}$ are $\lambda_{1}=-\mathrm{r}_{1}, \lambda_{2} \frac{\mathrm{bs}_{1}+\beta \mathrm{r}_{1}}{\mathrm{~b}}$. Since $\lambda_{1}<0$ and $\lambda_{2}>0$, the equilibrium point $\mathrm{E}_{2}$ of model

(2) is a saddle point. The Jacobian matrix of model

(2) at $E_{3}$ is

$J_{3}=\left(\begin{array}{cc}\frac{e r_{1}+\alpha s_{1}}{e} & 0 \\ \frac{\beta s_{1}}{e} & -s_{1}\end{array}\right)$

The eigenvalues of $\mathrm{J}_{3}$ are $\lambda_{1}=\frac{\mathrm{er}_{1}+\alpha \mathrm{s}_{1}}{\mathrm{e}}, \lambda_{2}=-\mathrm{s}_{1}$. Since $\lambda_{1}>0$ and $\lambda_{2}<0$, the equilibrium point $E_{3}$ of model (2) is a saddle point. The Jacobian matrix of model (2) at $\mathrm{E}^{*}$ is

$J^{*}=\left(\begin{array}{cc}-b x^{*} & \alpha x^{*} \\ \beta y^{*} & -e y^{*}\end{array}\right)$,

and we have the characteristic equation

$\lambda^{2}+\left(b x^{*}+e y^{*}\right) \lambda+\left(b e x^{*} y^{*}-\alpha \beta x^{*} y^{*}\right)$

This has the roots

$\lambda_{1,2}=\frac{-\left(b x^{*}+e y^{*}\right)}{2} \pm \frac{\sqrt{\left(b x^{*}+e y^{*}\right)^{2}-4\left(b e x^{*} y^{*}-\alpha \beta x^{*} y^{*}\right)}}{2}$.

Under the condition be $-\alpha \beta>0$, then $\left(b^{*}+\right.$ $\left.\mathrm{ey}^{*}\right)^{2}-4\left(\mathrm{bex}^{*} \mathrm{y}^{*}-\alpha \beta \mathrm{x}^{*} \mathrm{y}^{*}\right)>0$ and (be $\alpha \beta) x^{*} y^{*}>0$. Thus, both eigenvalues have negative real roots. Since $\lambda_{1}<0$ and $\lambda_{2}<0$, the unique positive equilibrium point $\mathrm{E}^{*}$ of model (2) is locally asymptotically stable.

\section{Global stability}

The intention in this section is to investigate the global stability of the unique positive equilibrium point $E^{*}=\left(x^{*}, y^{*}\right)$ in model (2) by constructing a suitable Lyapunov function when the conditions $r_{1}=$ $r-h_{x}>0, s_{1}=s-h_{y}>0$ and $b e-\alpha \beta>0$ are satisfied. In order to prove the global stability of the equilibrium point $E^{*}=\left(x^{*}, y^{*}\right)$ of model (2), we analyze the associated linearization model with perturbation $u$ and $v$. Let $u=x-x^{*}$ and $v=y-y^{*}$ and substitute into model (2) to yield

$\frac{d u}{d t}=r_{1}\left(u+x^{*}\right)-b\left(u+x^{*}\right)^{2}+\alpha\left(u+x^{*}\right)\left(v+y^{*}\right)$,
$\frac{d v}{d t}=s_{1}\left(v+y^{*}\right)-e\left(v+y^{*}\right)^{2}+\beta\left(u+x^{*}\right)\left(v+y^{*}\right)$,

or

$\frac{d u}{d t}=r_{1} u+r_{1} x^{*}-b u^{2}-2 b x^{*} u-b\left(x^{*}\right)^{2}+\alpha u v+\alpha y^{*} u+$

$\alpha x^{*} v+\alpha x^{*} y^{*}$

$\frac{d v}{d t}=s_{1} v+s_{1} y^{*}-e v^{2}-2 e y^{*} v-e\left(y^{*}\right)^{2}+\beta u v+\beta y^{*} u+$ $\beta x^{*} v+\beta x^{*} y^{*}$.

After simplifying and neglecting the product terms, we obtain the linearized model

$\frac{d u}{d t}=-b x^{*} u+\alpha x^{*} v$
$\frac{d v}{d t}=-e y^{*} v+\beta y^{*} u$
The corresponding characteristic equation is obtained from

$\left|\begin{array}{cc}-b x^{*}-\lambda & \alpha x^{*} \\ \beta y^{*} & -e y^{*}-\lambda\end{array}\right|=0$

which is given by

$\lambda^{2}+a_{00}+a_{11} \lambda=0$,

where

$a_{00}=(b e-\alpha \beta) x^{*} y^{*}>0$,

$a_{11}=b x^{*}+e y^{*}>0$.

We define a function by

$V(u, v)=A u^{2}+B u v+C v^{2}$,

where (Eqs. 6-9)

$A=\frac{\left(\beta y^{*}\right)^{2}+\left(e y^{*}\right)^{2}+(b e-\alpha \beta) x^{*} y^{*}}{2 D}$,

$B=\frac{b \beta x^{*} y^{*}+e \alpha x^{*} y^{*}}{D}$,

$C=\frac{\left(b x^{*}\right)^{2}+\left(\alpha x^{*}\right)^{2}+(b e-\alpha \beta) x^{*} y^{*}}{2 D}$ and

$D=a_{00} a_{11}=\left(b x^{*}+e y^{*}\right)(b e-\alpha \beta) x^{*} y^{*}$.

This function is certainly continuous with continuous first partial derivatives. Next, we want to check whether the function $V(u, v)$ is positive or negative definite. Function $V(u, v)$ is positive definite if, and only if $A>0$ and $4 A C-B^{2}>0$, and is negative definite if, and only if $A<0$ and $4 A C-$ $B^{2}>0$. From (6), it is clear that $A>0$ since the condition for the existence of positive equilibrium point is $b e-\alpha \beta>0$. Then,

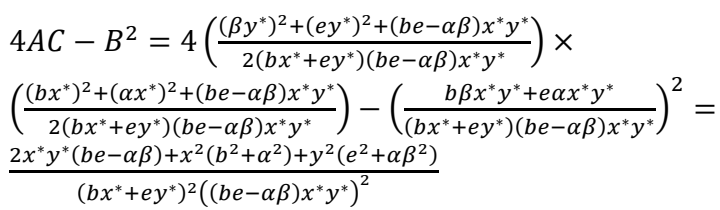

Since $\quad b e-\alpha \beta>0$, then $4 A C-B^{2}>0$. Therefore, the function $V(u, v)$ is positive definite. The chosen function $V(u, v)$ satisfies the first two requirements of a Lyapunov function.

For the final requirement that the time derivative of $V(u, v)$ must be negative definite, by using the linearized system (3), we have

$\frac{\partial V}{\partial u} \frac{d u}{d t}+\frac{\partial V}{\partial v} \frac{d v}{d t}=(2 A u+B v)\left(-b x^{*} u+\alpha x^{*} v\right)+$

$(B u+2 C v)\left(-e y^{*} v+\beta y^{*} u\right)$.

Substituting the values of $A, B, C$ and $D$ from (6), (7), (8) and (9) in (10) we get

$\frac{\partial V}{\partial u} \frac{d u}{d t}+\frac{\partial V}{\partial v} \frac{d v}{d t}=\frac{(b e-\alpha \beta)\left(b x^{*}+e y^{*}\right)\left(-u^{2}-v^{2}\right)}{\left(b x^{*}+e y^{*}\right)(b e-\alpha \beta)}=-\left(u^{2}+v^{2}\right)$

which is clearly negative definite. So $V(u, v)$ is a Lyapunov function for the linearized system (3).

Next, we proceed to prove that $V(u, v)$ is also a Lyapunov function for the nonlinear system (2). 

by

Let $F_{1}$ and $F_{2}$ be two functions of $x$ and $y$ defined

$F_{1}(x, y)=r_{1} x-b x^{2}+\alpha x y$,

$F_{2}(x, y)=s_{1} y-e y^{2}+\beta x y$.

We have to show that $\frac{\partial V}{\partial u} F_{1}+\frac{\partial V}{\partial v} F_{2}$ is negative definite. By letting $u=x-x^{*}$ and $v=y-y^{*}$ in (2), we have

$\frac{d u}{d t}=r_{1} u+r_{1} x^{*}-b u^{2}-2 b x^{*} u-b\left(x^{*}\right)^{2}+\alpha u v+\alpha y^{*} u+$ $\alpha x^{*} v+\alpha x^{*} y^{*}$.

After simplifying, we have

$\frac{d u}{d t}=\left(r_{1} x^{*}-b\left(x^{*}\right)^{2}+\alpha x^{*} y^{*}\right)+\left(r_{1}-b x^{*}+\alpha y^{*}\right) u-$

$b x^{*} u+\alpha x^{*} v-b u^{2}+\alpha u v$.

The first and second terms of $\frac{d u}{d t}$ are equal to zero at the equilibrium point $\left(x^{*}, y^{*}\right)$. Thus, we have

$\frac{d u}{d t}=-b x^{*} u+\alpha x^{*} v-b u^{2}+\alpha u v=-b x^{*} u+\alpha x^{*} v+$

$f_{1}(u, v)=F_{1}(u, v)$,

where

$f_{1}(u, v)=-b u^{2}+\alpha u v$.

Similiarly, we obtain

$\frac{d v}{d t}=s_{1} v+s_{1} y^{*}-e v^{2}-2 e y^{*} v-e\left(y^{*}\right)^{2}+\beta u v+\beta y^{*} u+$ $\beta x^{*} v+\beta x^{*} y^{*}$.

After simplifying, we have

$\frac{d v}{d t}=\left(s_{1} y^{*}-e\left(y^{*}\right)^{2}+\beta x^{*} y^{*}\right)+\left(s_{1}-e y^{*}+\beta x^{*}\right) v-$ $e y^{*} v+\beta y^{*} u-e v^{2}+\beta u v$.

The first and second terms of $\frac{d v}{d t}$ are equal to zero at the equilibrium point $\left(x^{*}, y^{*}\right)$. Thus, we have

$\frac{d v}{d t}=-e y^{*} v+\beta y^{*} u-e v^{2}+\beta u v=-e y^{*} v+\beta y^{*} u+$

$f_{2}(u, v)=F_{2}(u, v)$,

where

$f_{2}(u, v)=-e v^{2}+\beta u v$.

From (5), we have

$\frac{\partial V}{\partial u}=2 A u+B v$,

and

$\frac{\partial V}{\partial v}=B u+2 C v$

Hence,

$\frac{\partial V}{\partial u} F_{1}+\frac{\partial V}{\partial v} F_{2}=(2 A u+B v)\left(-b x^{*} u+\alpha x^{*} v+f_{1}(u, v)\right)+$

$(B u+2 C v)\left(-e y^{*} v+\beta y^{*} u+f_{2}(u, v)\right)=(2 A u+$

$B v)\left(-b x^{*} u+\alpha x^{*} v\right)+(B u+2 C v)\left(-e y^{*} v+\beta y^{*} u\right)+$

$(2 A u+B v) f_{1}(u, v)+(B u+2 C v) f_{2}(u, v)$.
From (10) and (11), we obtain

$\frac{\partial V}{\partial u} F_{1}+\frac{\partial V}{\partial v} F_{2}=-\left(u^{2}+v^{2}\right)+(2 A u+B v) f_{1}(u, v)+$

$(B u+2 C v) f_{2}(u, v)$.

Introducing polar co-ordinates $u=R \cos \theta, v=$ $R \sin \theta$, (14) can be written as

$\frac{\partial V}{\partial u} F_{1}+\frac{\partial V}{\partial v} F_{2}=-R^{2}+R\left[(2 A \cos \theta+B \sin \theta) f_{1}(u, v)\right]+$

$R\left[B(\cos \theta+2 C \sin \theta) f_{2}(u, v)\right]$.

Let us denote the largest of the numbers $|2 A|,|B|$ and $|2 C|$ by $K$. Our assumptions imply that $\left|f_{1}(u, v)\right|<\frac{R}{8 K}$ and $\left|f_{2}(u, v)\right|<\frac{R}{8 K}$ for all sufficiently small $R>0$, so that

$\frac{\partial V}{\partial u} F_{1}+\frac{\partial V}{\partial v} F_{2}<-R^{2}+R\left((2 A \cos \theta+B \sin \theta) \frac{R}{8 K}\right)+$

$R\left(B(\cos \theta+2 C \sin \theta) \frac{R}{8 K}\right)$

$<-R^{2}+\frac{R^{2}}{8 K}((|2 A|+|B|) \cos \theta+(|B|+|2 C|) \sin \theta)$

$<-R^{2}+\frac{R^{2}}{8 K}(2 K \cos \theta+2 K \sin \theta)$

$<-R^{2}+\frac{R^{2}}{8 K}(4 K)$.

This implies

$\frac{\partial V}{\partial u} F_{1}+\frac{\partial V}{\partial v} F_{2}<-R^{2}+\frac{4 K R^{2}}{8 K}=-\frac{R^{2}}{2}<0$.

Thus, $V(u, v)$ is a positive definite function with the property that $\frac{\partial V}{\partial u} F_{1}+\frac{\partial V}{\partial v} F_{2}$ is negative definite. So, $V(u, v)$ is a Lyapunov function for the nonlinear system (2). Therefore, the unique positive equilibrium point $E^{*}=\left(x^{*}, y^{*}\right)$ of model (2) is globally asymptotically stable.

\section{Numerical simulation}

Now, we carry out numerical simulation to investigate the dynamic behavior of the system (1) about the equilibrium point $E^{*}=\left(x^{*}, y^{*}\right)$. Consider the following system of wildebeest and zebra without the presence of lion as proposed in Fay and Greeff (2006) by incorporating proportional harvesting to illustrate the theory discussed previously

$\frac{d x}{d t}=0.405 x-0.03375 x^{2}+0.015 x y-0.2 x$,

$\frac{d y}{d t}=0.34 y-0.02833 y^{2}+0.020 x y-0.1 y$,

where $x$ and $y$ represent the population size of wildebeest and zebra respectively (both measured in thousands). Harvesting refers to the process of cropping the species, i.e. removing the species to a new location in order to avoid overcrowding.

Comparing model (16) with model (1), we have $r=0.405, s=0.34, b=0.03375, e=0.02833, \alpha=$ 0.015 and $\beta=0.020$. The harvesting rate $h_{x}=0.2$ and $h_{y}=0.1$ are chosen to satisfied the conditions $0<h_{x}<r$ and $0<h_{y}<s$. 
Let $r_{1}=r-h_{x}$ and $s_{1}=s-h_{y}$, so that model (16) can be written as follow

$$
\begin{aligned}
& \frac{d x}{d t}=0.205 x-0.03375 x^{2}+0.015 x y \\
& \frac{d y}{d t}=0.24 y-0.02833 y^{2}+0.020 x y
\end{aligned}
$$

Comparing model (17) with model (2), we have $r_{1}=0.205, b=0.03375, \alpha=0.015, s_{1}=0.24, e=$ 0.02833 and $\beta=0.020$. With these parameters, the conditions be $-\alpha \beta>0, r_{1}=r-h_{x}>0$ and $s_{1}=$ $s-h_{y}>0$ for the existence of the unique positive equilibrium point are satisfied. The unique positive equilibrium point is $E^{*}=(14.3365,18.5905)$. The eigenvalues associated with this equilibrium point are -0.2217 and -0.7889 . This means that $E^{*}$ is locally asymptotically stable.

We would like to determine the stability of the model (16) where the equilibrium point is $\left(x^{*}, y^{*}\right)=$ $(14.3365,18.5905)$. In Fig. 1, the equilibrium point $(14.3365,18.5905)$ is a stable sink. This means that solution trajectories tend to the equilibrium point without oscillation. The population dynamics of wildebeest and zebra can be analyzed separately with respect to time. From Fig. 2 and Fig. 3, we can see that both wildebeest and zebra populations converge in finite time to their equilibrium values $x^{*}=14.3365$ and $y^{*}=18.5905$ from initial population $x(0)=14$ and $y(0)=17$. As shown in Figs. 1, 2, and 3 , the unique positive equilibrium point $E^{*}=(14.3365,18.5905)$ is globally asymptotically stable and all the two species persist.

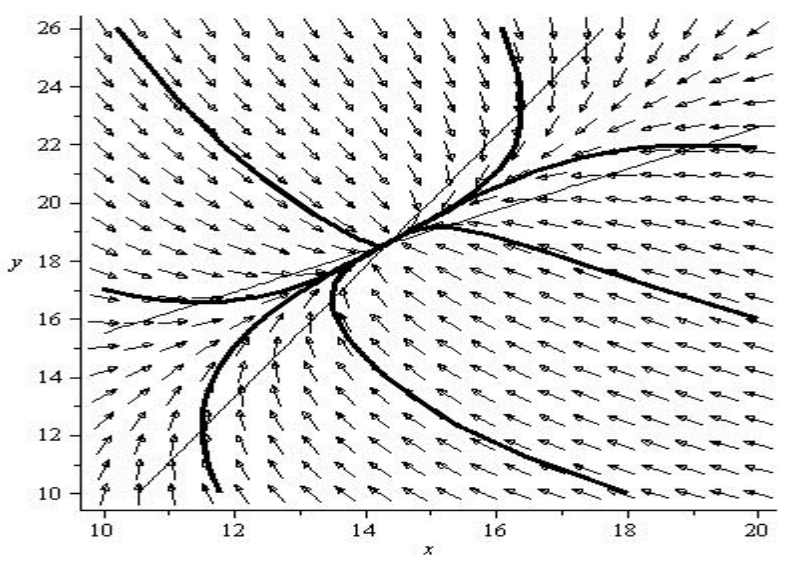

Fig. 1: Phase portrait of $(x(t), y(t))$

\section{Conclusion}

This study considered a two-species mutualism model with proportional harvesting. A suitable Lyapunov function was successfully constructed in order to prove the global stability of the unique positive equilibrium point when the conditions $r_{1}=$ $r-h_{x}>0, s_{1}=s-h_{y}>0$ and $b e-\alpha \beta>0$ are satisfied. From the numerical analysis, it is observed that both the species persist. As seen from Fig. 1-Fig. 3 , both the wildebeest and zebra populations tend toward the equilibrium point $(14.3365,18.5905)$.

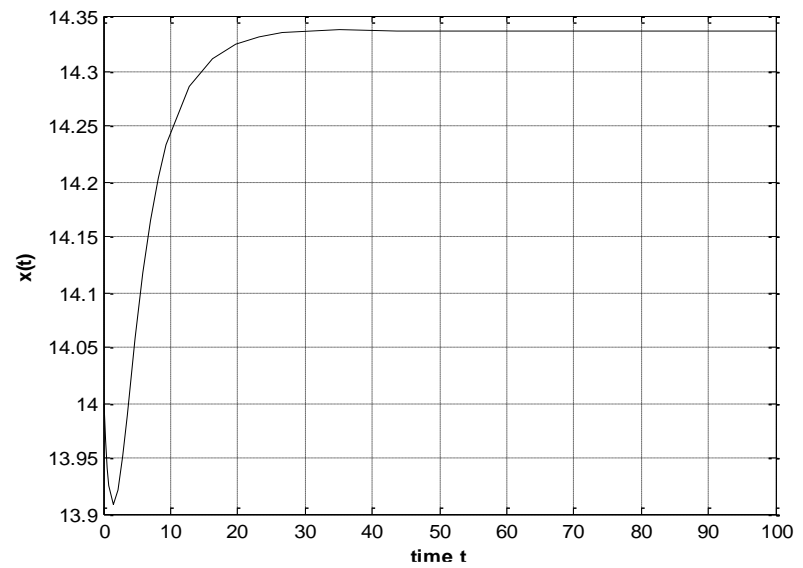

Fig. 2: Trajectory of $x(t)$ at $h_{x}=0.2$ and $x(0)=14$

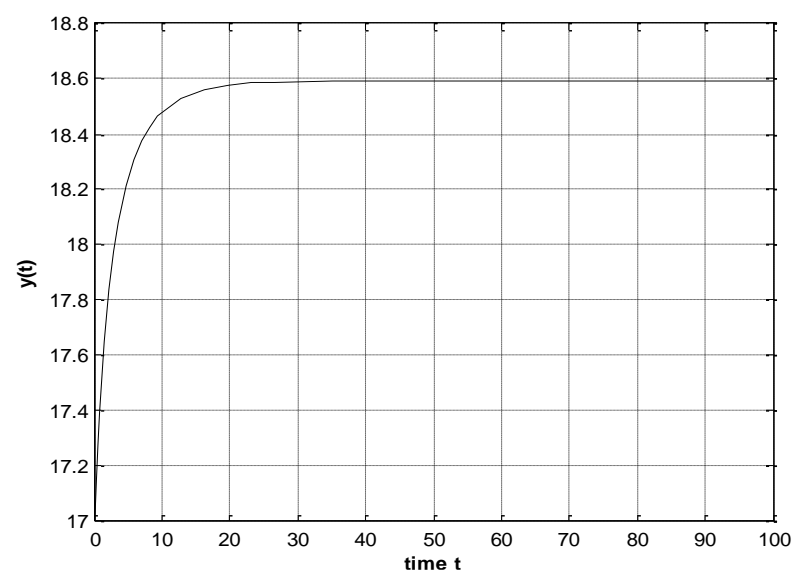

Fig. 3: Trajectory of $y(t)$ at $h_{y}=0.1$ and $y(0)=17$

There is still a tremendous amount of work to be done in this area. For example, it is interesting to prove the global stability of two or more species mutualism models having both delay and harvesting function in a real life situation. Previous researchers have studied models with either delay (Hsu and Ho, 2006; León, 2015; Saito, 2002) or harvesting (León, 2012) but as far as we know, no research has been done for models having both components.

The literature on the stability of the mutualism model is not as much as the literature concerning the stability of the predator-prey and competition models. Therefore, the findings of this study may contribute to a better understanding of the population dynamics of mutualism model particularly in the use of Lyapunov function to determine the global stability when the model incorporates harvesting function. Continuous effort in this area can add to the body of knowledge and benefits the society especially regards to the conservation of species within the ecosystem.

\section{References}

Benz R (2000). Ecology and evolution: Islands of change. NSTA Press, Virginia, USA.

Boyce WE and DiPrima RC (1992). Elementary differential equations and boundary value problems. John Wiley and Sons, Hoboken, USA.

Cheng KS, Hsu SB, and Lin SS (1981). Some results on global stability of a predator-prey system. Journal of Mathematical Biology, 12(1): 115-126. 
Do KD and Pan J (2009). Control of ships and underwater vehicles. Springer Science and Business Media, Berlin, Germany.

Fay TH and Greeff JC (2006). Lion, wildebeest and zebra: a predator-prey model. Ecological Modeling, 196(1): 237-244.

Georgescu P, Zhang H, and Maxin D (2016). The global stability of coexisting equilibria for three models of mutualism. Mathematical Biosciences and Engineering, 13(1): 101-118.

Goh BS (1979). Stability in models of mutualism. The American Naturist, 113(2): 261-275.

Haberman R (1998). Mathematical models: Mechanical vibrations, population dynamics and traffic flow. Society for Industrial and Applied Mathematics, Pennsylvania, USA.

Hsu FC and Ho CP (2006). Global stability for the lotka-volterra mutualistic system with time delay. Tunghai Science, 8: 81107.

Janzen DH (1985). The natural history of mutualisms. In: Boucher DL (Ed.), The biology of mutualism: Ecology and evolution: 40-99. Oxford University Press, New York, USA

Kot M (2001). Elements of mathematical ecology. The Press Syndicate of the University of Cambridge, Cambridge University Press, Cambridge, UK.
León CVD (2012). Lyapunov function for two-species cooperative systems. Applied Mathematics and Computation, 219(5): 2493-2497.

León CVD (2015). Lyapunov functional for global stability of lotkavolterra cooperative systems with discrete delays. Abstraction and Application, 12: 42-50.

Morin PJ (2011). Community ecology. John Wiley and Sons, Hoboken, USA

Ouncharoen R, Pinjai S, Dumrongpokaphan T, and Lenbury Y (2012). Global stability analysis of predator-prey model with harvesting and delay. Thai Journal of Mathematics, 8(3): 589605.

Reddy BR, Narayan KL, and Pattabhiramacharyulu NC (2011). On global stability of two mutually interacting species with limited resources for both the species. International Journal of Contemporary Mathematical Sciences, 6(9): 401-407.

Rockwood LL (2015). Introduction to population ecology. John Wiley and Sons, Hoboken, USA.

Saito Y (2002). The necessary and sufficient condition for global stability of a lotka-volterra cooperative or competition system with delays. Journal of Mathematical Analysis and Applications, 268(1): 109-124.

Starr C, Taggart R, Evers C, and Starr L (2015). Biology: The unity and diversity of life. Nelson Education, Toronto, Canada. 